\title{
Feedback correction of injection errors using digital signal-processing techniques
}

\author{
N. S. Sereno* and F. R. Lenkszus ${ }^{\dagger}$ \\ Argonne National Laboratory, Advanced Photon Source, 9700 South Cass Avenue, Argonne, Illinois 60439, USA
}

(Received 10 July 2006; published 22 January 2007)

\begin{abstract}
Efficient transfer of electron beams from one accelerator to another is important for 3rd-generation light sources that operate using top-up. In top-up mode, a constant amount of charge is injected at regular intervals into the storage ring to replenish beam lost primarily due to Touschek scattering. Top-up therefore requires that the complex of injector accelerators that fill the storage ring transport beam with a minimum amount of loss. Injection can be a source of significant beam loss if not carefully controlled. In this note we describe a method of processing injection transient signals produced by beam-position monitors and using the processed data in feedback. Feedback control using the technique described here has been incorporated in the Advanced Photon Source (APS) booster synchrotron to correct injection transients.
\end{abstract}

DOI: 10.1103/PhysRevSTAB.10.012803

PACS numbers: 29.27.Eg

\section{INTRODUCTION}

Unwanted beam motion in a ring after injection can result from many sources including pulsed magnet mispowering, trajectory, energy, and phase errors. In general, each source will produce betatron and synchrotron oscillations which can be observed on beam-position monitors (BPM). For example, energy and phase errors in longitudinal phase space appear at a dispersive BPM as oscillations at the synchrotron frequency but have a phase difference of $90^{\circ}$. Similarly beam angle and displacement errors in transverse phase space produce oscillations at the fractional betatron frequency $90^{\circ}$ apart in phase. In the frequency domain, the injection transient signal at either the synchrotron or the betatron frequency contains information about each type of longitudinal or transverse error. A feedback process can use this frequency-domain information to set actuators that act to zero the injection errors. The analysis presented here is general and can be applied as long as the injection transient oscillation can be cleanly detected.

\section{INJECTION TRANSIENT ANALYSIS IN THE TIME AND FREQUENCY DOMAINS}

In this section we derive the frequency-domain expressions that depend linearly on the various injection errors that can cause transients at a dispersive BPM. In the time domain, it is the amplitude and phase of the longitudinal or transverse injection transient turn by turn that depend on a particular kind of error. If one looks at the same transient in the frequency domain, one finds the amplitude and phase at either the synchrotron or betatron sideband frequency depend on the various injection errors though not linearly. The following analysis will show that the quantities that vary linearly with longitudinal and transverse injection

\footnotetext{
*Electronic address: sereno@aps.anl.gov

†Electronic address: frl@aps.anl.gov
}

errors are the real and imaginary parts of the injection transient in the frequency domain at either the synchrotron or betatron sideband.

The injection transient analysis begins in the time domain. In general, a given pair of conjugate phase-space coordinates at turn $n$ are computed according to the matrix equation,

$$
\left[\begin{array}{c}
\zeta \\
\zeta^{\prime}
\end{array}\right]_{n}=\mathbf{M}\left[\begin{array}{l}
\zeta \\
\zeta^{\prime}
\end{array}\right]_{n-1},
$$

where the motion is implicitly assumed to be linear. The coordinates after $n$ turns can be found by $n$ successive applications of the matrix $\mathbf{M}$ to the initial coordinates. The resulting matrix after $n$ turns can be parametrized according to

$$
\begin{gathered}
\mathbf{M}^{n}=\mathbf{I} \cos \left(n \omega_{\zeta} T_{\circ}\right)+\mathbf{J} \sin \left(n \omega_{\zeta} T_{\circ}\right) \\
\mathbf{J}=\left[\begin{array}{cc}
\alpha_{\zeta} & \beta_{\zeta} \\
-\gamma_{\zeta} & -\alpha_{\zeta}
\end{array}\right],
\end{gathered}
$$

where $\mathbf{I}$ is the identity matrix and the $\mathbf{J}$ matrix contains the Twiss parameters and $\zeta$ denotes the horizontal, vertical, or longitudinal planes [1]. In Eq. (2), $\omega_{\zeta}$ is the betatron or synchrotron frequency, and $T_{\circ}=2 \pi / \omega_{\circ}$ is the ring revolution time. The usual condition

$$
|\operatorname{tr} \mathbf{M}| \leq 2
$$

is assumed satisfied so that the motion is bounded and stable. In the case of longitudinal motion, the conjugate coordinates are $\zeta=\Delta \phi$ and $\zeta^{\prime}=\Delta E$, where these quantities determine the particle longitudinal position relative to the phase and energy of the reference particle. The longitudinal Twiss parameters are given by

$$
\alpha_{s}=0
$$




$$
\beta_{s}=\frac{1}{\gamma_{s}}=\frac{h|\eta|}{\beta^{2} E \nu_{s}}
$$

where $s$ is used to denote the longitudinal plane, $h$ is the $\mathrm{rf}$ system harmonic number, $E$ is the beam energy, $\eta$ is the slip factor, $\beta$ is the relativistic factor, and

$$
\nu_{s} \equiv \frac{\omega_{s}}{\omega_{\circ}}=\sqrt{\frac{h\left|\eta \cos \phi_{s}\right|}{2 \pi \beta^{2}} \frac{e V}{E}}
$$

is the synchrotron tune, $\phi_{s}$ is the synchronous phase, $\mathrm{eV}$ is the rf system gap voltage [2], and $E$ is the synchronous particle energy. Longitudinal stability requires that $\eta \cos \phi_{s}<0$. Time and energy can also be used to describe longitudinal motion and are equivalent to phase and energy. For the following development we use phase and energy as the longitudinal coordinates for convenience.

The previous results can now be used to develop an analysis of beam transient signals observed at a BPM after injection of the beam into the ring. In general, the transverse beam position at a ring BPM in the dispersive plane (usually the horizontal plane) at turn $n$ is

$$
\xi(n)=\xi\left(n, \omega_{s}\right)+\xi\left(n, \omega_{\xi}\right),
$$

where $\xi\left(n, \omega_{s}\right)=\eta \Delta E / E$ is the transverse displacement due to synchrotron motion, $\xi\left(n, \omega_{\xi}\right)$ is the transverse displacement due to betatron motion, $\omega_{s}$ is the synchrotron frequency, $\omega_{\xi}$ is the betatron frequency, and $\xi$ is used to denote either the horizontal or vertical plane. The two types of motion are distinguished by their respective oscillation frequencies. In the nondispersive plane (usually the vertical plane), there is no contribution due to synchrotron motion since the dispersion is zero. Betatron transient motion at injection is seen at the fractional part of betatron tune and is much faster than the corresponding synchrotron motion. Buried within each type of motion is information about the particular combination of errors that caused the motion. Equations (2), (3), (5), and (6) imply after rearranging terms,

$$
\xi\left(n, \omega_{s}\right)=\eta \frac{\Delta E_{\circ}}{E} \cos \left(n \omega_{s} T_{\circ}\right)-\eta \frac{\Delta \phi_{\circ}}{E \beta_{s}} \sin \left(n \omega_{s} T_{\circ}\right)
$$

$$
\begin{aligned}
\xi\left(n, \omega_{\xi}\right)= & \xi_{\circ} \cos \left(n \omega_{\xi} T_{\circ}\right)+\left\{\alpha_{\xi} \xi_{\circ}+\beta_{\xi} \xi_{\circ}^{\prime}\right\} \\
& \times \sin \left(n \omega_{\xi} T_{\circ}\right),
\end{aligned}
$$

where $\eta$ is the dispersion at the BPM, $\Delta E_{\circ}$ is the injected energy error, $\Delta \phi_{\mathrm{o}}$ is the injected phase error, and $\beta_{s}$ is the longitudinal beta function given by Eq. (6).

Equations (9) and (10) indicate that the signature of the various injection errors is cosinelike and sinelike oscillations in the time domain. Energy and phase errors are seen to create cosinelike and sinelike, synchrotron oscillations, respectively. Transverse position errors on the other hand cause both cosinelike and sinelike betatron oscillations if $\alpha_{\xi}$ is nonzero. Transverse angle errors are seen to cause only sinelike betatron oscillations. In the frequency domain, the amplitude at the synchrotron and betatron oscillation frequencies depends on the magnitude of the various injection errors. In Appendix A, Eqs. (9) and (10) are cast in the frequency domain using the discrete Fourier transform (DFT) [3].

When cast in the frequency domain, the real and imaginary parts of Eqs. (9) and (10) only depend on the real and imaginary parts of the sine and cosine frequency-domain oscillation terms computed in Appendix A. The real and imaginary parts of the transverse position at the betatron and synchrotron frequencies therefore depend linearly on the corresponding phase-space coordinate errors. The synchrotron and betatron response matrices can be defined as

$$
\begin{aligned}
& {\left[\begin{array}{l}
\operatorname{Re}\left[\Xi\left(\omega_{s}\right)\right] \\
\operatorname{Im}\left[\Xi\left(\omega_{s}\right)\right]
\end{array}\right]=\mathbf{R}\left(\omega_{s}\right)\left[\begin{array}{c}
\Delta \phi \\
\Delta E
\end{array}\right]} \\
& {\left[\begin{array}{l}
\operatorname{Re}\left[\Xi\left(\omega_{\xi}\right)\right] \\
\operatorname{Im}\left[\Xi\left(\omega_{\xi}\right)\right]
\end{array}\right]=\mathbf{R}\left(\omega_{\xi}\right)\left[\begin{array}{l}
\xi_{0} \\
\xi_{\circ}^{\prime}
\end{array}\right],}
\end{aligned}
$$

where the matrices are given by

$$
\begin{gathered}
\mathbf{R}\left(\omega_{s}\right)=\frac{\eta}{E}\left[\begin{array}{ll}
\operatorname{Re}\left[C\left(\omega_{s}\right)\right] & -\operatorname{Re}\left[S\left(\omega_{s}\right)\right] / \beta_{s} \\
\operatorname{Im}\left[C\left(\omega_{s}\right)\right] & -\operatorname{Im}\left[S\left(\omega_{s}\right)\right] / \beta_{s}
\end{array}\right] \\
\mathbf{R}\left(\omega_{\xi}\right)=\left[\begin{array}{cc}
\operatorname{Re}\left[C\left(\omega_{\xi}\right)\right] & \\
+\alpha_{\xi} \operatorname{Re}\left[S\left(\omega_{\xi}\right)\right] & \beta_{\xi} \operatorname{Re}\left[S\left(\omega_{\xi}\right)\right] \\
\operatorname{Im}\left[C\left(\omega_{\xi}\right)\right] & \\
+\alpha_{\xi} \operatorname{Im}\left[S\left(\omega_{\xi}\right)\right] & \beta_{\xi} \operatorname{Im}\left[S\left(\omega_{\xi}\right)\right]
\end{array}\right],
\end{gathered}
$$

and the symbols $C\left(\omega_{(s, \xi)}\right)$ and $S\left(\omega_{(s, \xi)}\right)$ are the complex cosinelike $\left[\cos \left(n \omega_{(s, \xi)} T_{\circ}\right)\right]$ and sinelike $\left[\sin \left(n \omega_{(s, \xi)} T_{\circ}\right)\right]$ oscillations in the frequency domain, $\Xi\left(\omega_{(s, \xi)}\right)$ is the DFT of the betatron or synchrotron oscillation $\xi\left(n, \omega_{(s, \xi)}\right)$ defined by Eqs. (9) and (10), and $\operatorname{Re}($ ) and $\operatorname{Im}($ ) denote taking the real and imaginary parts of a complex quantity.

Each matrix $\mathbf{R}\left(\omega_{s}\right)$ or $\mathbf{R}\left(\omega_{\xi}\right)$ when inverted is used in a feedback loop to correct either betatron or synchrotron transients. The feedback loop uses the real and imaginary parts of the transverse position DFT at either the betatron or synchrotron frequencies as the "readback" variables, and the "actuator" variables can be correctors or pulsed magnets for the case of betatron transient correction and beam energy and rf phase for synchrotron transient correction. Each response matrix element in Eqs. (13) and (14) is rather complicated analytically and will also depend on the time-domain window function used to process the beam history data. In our implementation, the response matrix elements are measured once by changing the actuator variables that affect each phase-space coordinate and measuring the change in the real and imaginary parts of the DFT. Our implementation also uses the fast Fourier transform (FFT) algorithm to process the transient oscillation, 
since it is equivalent to but much faster computationally than the DFT algorithm.

\section{APS INJECTOR LAYOUT AND OPERATION}

Figure 1 shows a schematic layout of the Advanced Photon Source (APS) injector accelerators. The electron beam originates in the thermionic rf gun and is injected into the linac. The linac accelerates the beam to $325 \mathrm{MeV}$ where it is injected into the particle accumulator ring (PAR). The PAR accumulates up to five linac pulses and damps them into a single rf bucket containing up to $5 \mathrm{nC}$ of charge. The PAR beam is injected into a single booster bucket, where it is brought on-axis by the booster-injection septum and kicker. The booster accelerates the beam in $223 \mathrm{~ms}$ linearly from $325 \mathrm{MeV}$ to $7 \mathrm{GeV}$ and repeats this acceleration cycle at $2 \mathrm{~Hz}$. The beam is injected into the booster "on the fly" [4] as the magnetic field is linearly ramped to its final $7 \mathrm{GeV}$ value (there is no constant field part of the booster cycle when the beam is injected). The $7 \mathrm{GeV}$ beam is then transported to the APS storage ring and injected.

In the APS "top-up" operation mode, a single pulse of approximately $2 \mathrm{nC}$ of charge is injected into the storage ring every two minutes to replace charge lost due to Touschek and gas scattering. Top-up represents the most demanding operations mode since the linac, PAR, and booster must provide beam to the storage ring on a 24hour basis. Injection transients in the booster can arise primarily from booster magnet and PAR rf phase drift. These transients can cause loss of top-up pulses if they become too large, so feedback correction is used to suppress transients as they arise.

\section{INJECTION TRANSIENT ACQUISITION AND PROCESSING IN THE APS BOOSTER SYNCHROTRON}

Injection transient feedback correction was implemented in the APS booster and is used during normal machine operations. Both horizontal and longitudinal injection transients are corrected. Horizontal transients are primarily due to slow drift in the linear magnet ramp waveforms over several $2 \mathrm{~Hz}$ cycles $[5,6]$. The ramp waveform drift is normally corrected by a separate feedback loop, which has a limited bandwidth and cannot correct for all sources of drift faster than about $0.3 \mathrm{~Hz}$. Horizontal transients can also arise due to thermal warm-up effects in the booster-injection septum supply. Longitudinal transients are also primarily generated by the dipole ramp drift, which causes an equivalent energy error. The rf phase errors arise from both the PAR and booster rf systems.

Figure 2 shows the booster BPM data-acquisition system used to acquire injection transients [7]. The four BPM button signals are bandpass filtered at the booster rf frequency $(352 \mathrm{MHz})$ and subtracted using $180^{\circ}$ hybrids to produce raw horizontal and vertical signals and a sum signal of all four buttons. The raw horizontal and vertical signals are then sent to the monopulse receiver, which performs electrical offset correction and normalizes the raw horizontal and vertical signals to the sum signal. The timing and control module allows normalized horizontal,

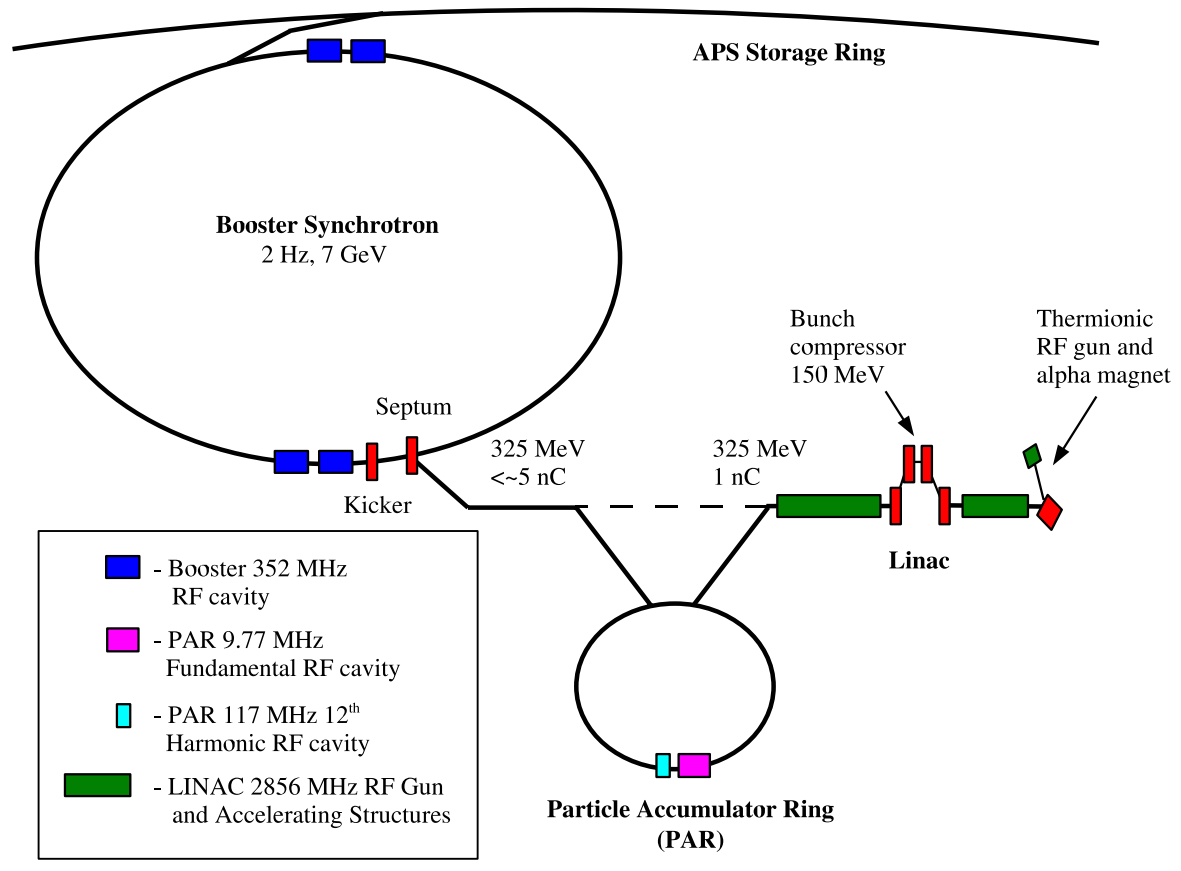

FIG. 1. (Color) Schematic diagram of the APS injector accelerators. 


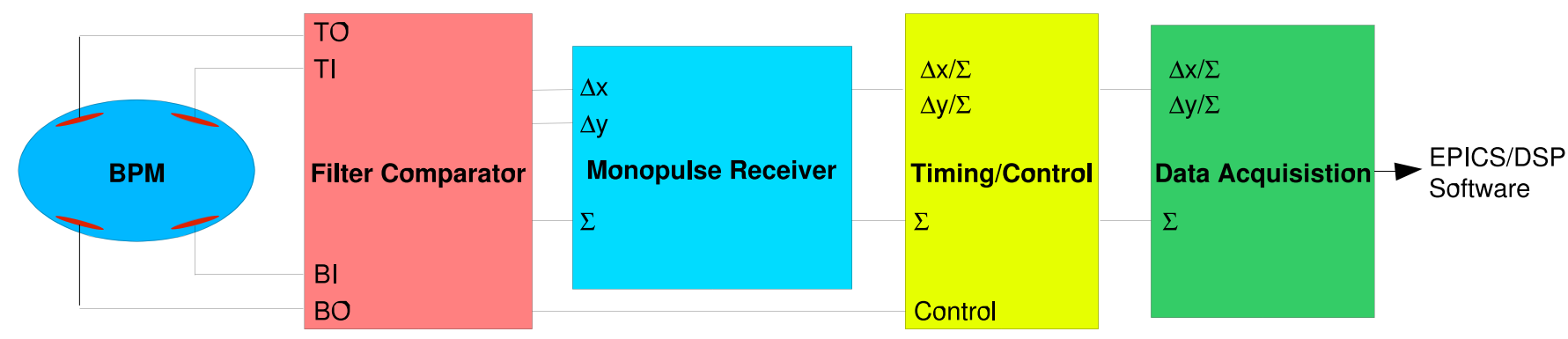

FIG. 2. (Color) Schematic diagram of the APS booster BPM signal conditioning/data-acquisition system.
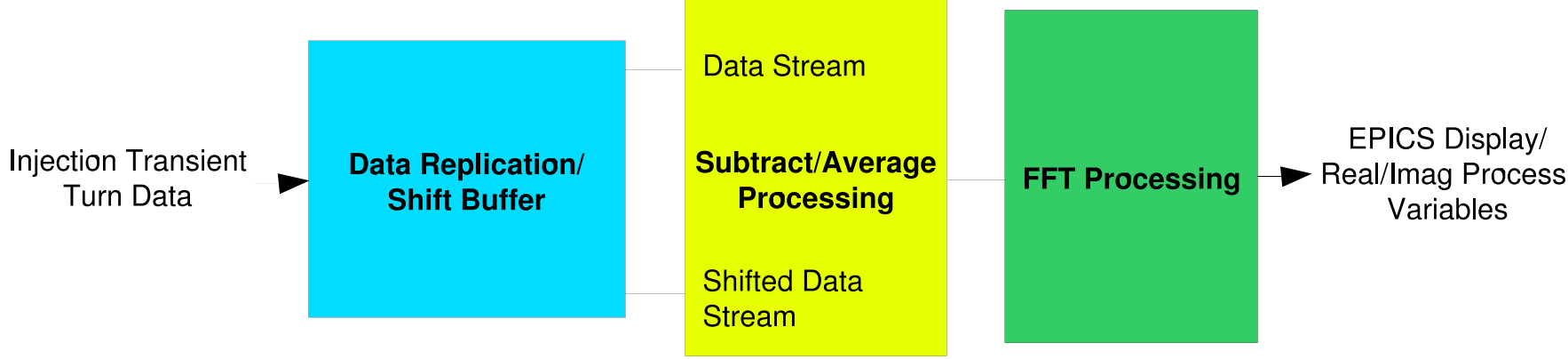

FIG. 3. (Color) Block diagram of injection transient signal processing.

vertical, or both signals to be transferred to the dataacquisition module. The data-acquisition module can accumulate up to 512 turns of either horizontal or vertical data or 256 turns of both horizontal and vertical data and send it to the EPICS control system for processing [8]. The data-acquisition module also specifies the turn that data accumulation begins. In this way, up to 512 turns of beamposition data can be accumulated at any point during the $223 \mathrm{~ms}$ it takes to accelerate the beam to $7 \mathrm{GeV}$.

For this application, injection transient data are acquired each 2-Hz ramp cycle. At injection 256 turns of data are acquired, which gives approximately 7 synchrotron oscillations and 64 horizontal betatron oscillations specified by the fractional part of the betatron frequency. Figure 3 shows the EPICS processing flow diagram of transients acquired during injection.

Transient data processing starts with replication and time shifting of the raw turn data as shown in Fig. 3. The raw and shifted data streams are subtracted and averaged to remove the orbit and BPM offset present in the data. Timedomain averaging of six subtracted and averaged waveforms is used in this application to reduce shot-to-shot noise. The processed data are then converted to the frequency domain using an FFT algorithm and a userselectable time-domain window. The window chosen in this application is the Blackman window. This window has the desirable property that the side lobes for a given signal are much reduced compared to other windows, such as the Hanning window. Sidelobe suppression is important since the real and imaginary parts of the FFT used in the feedback are taken at the frequency corresponding to the peak amplitude of the FFT, as shown in Fig. 4.

Figure 5 shows longitudinal injection transient signals in the time domain for a pure energy and a pure phase error using the signal-processing setup outlined in Figs. 2 and 3. One clearly sees the 90-degree phase difference between each type of error. Since the synchrotron frequency is slightly different cycle to cycle, the figure shows that the phase difference becomes greater than $90^{\circ}$ after two oscil-
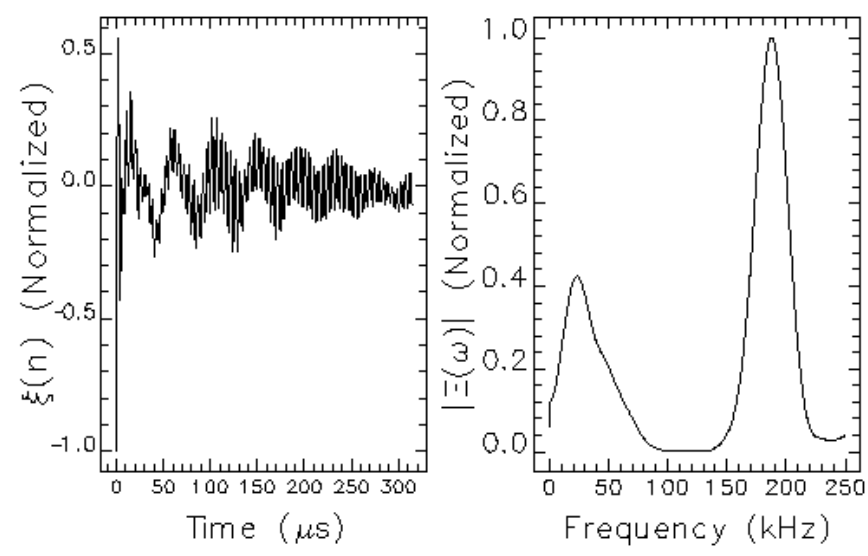

FIG. 4. Injection time-domain transient signal (left) along with FFT amplitude spectrum (right) obtained after processing the raw turn data according to Fig. 3. The time axis in the left figure was converted from turns $n$ to time in $\mu s$ using the revolution period of $1.228 \mu \mathrm{s}$ per turn. The synchrotron tune peak is at approximately $23 \mathrm{kHz}$, and the horizontal betatron tune is at $190 \mathrm{kHz}$. 


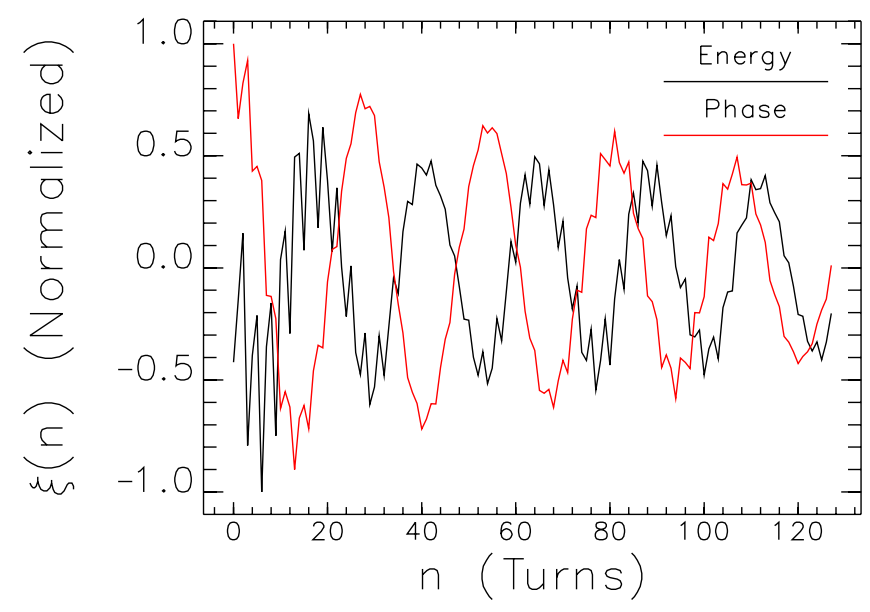

FIG. 5. (Color) Longitudinal injection transient signals in the time domain for a pure energy and a pure phase error using the BPM signal-processing setup outlined in Figs. 2 and 3. In the figure, time is represented as the number of turns after injection. One sees a 90-degree phase difference between each type of error.

lations. This additional phase shift does not present a problem operationally since the feedback uses averaging and only a percentage of the calculated correction. The energy error appears sinelike, and the phase error appears cosinelike in Fig. 5. Equation (9) indicates just the opposite. This discrepancy is an artifact of the time-shift subtraction and average processing used to remove orbit and BPM offset errors. Since the response matrix used in the feedback is measured rather than theoretical, it makes no difference how each error appears after processing since the crucial point is that the resulting phase and energy oscillations are orthogonal. Similarly the horizontal betatron oscillations produced from angle (kicker) and position offset errors (septum) are orthogonal, and this information is contained within the real and imaginary parts of the FFT at the horizontal betatron frequency.

\section{INJECTION TRANSIENT FEEDBACK IMPLEMENTATION IN THE APS BOOSTER SYNCHROTRON}

Injection transient feedback correction was implemented in the APS booster using the signal-processing techniques described in the last section and actuator variables specific to each type of injection transient error. In the case of longitudinal feedback, the actuators used are the rf phase of the booster, as well as the time at which the booster magnets start to ramp. The ramp "start time" controls the magnetic field of the booster magnets at the time the beam is injected. If the magnetic field is incorrect, the result is an effective energy error. The start ramp time variable is therefore used to control energy errors. For the case of horizontal betatron feedback, the actuator variables are the injection septum and injection kicker. The injection septum and kicker control, respectively, the amount of "cosinelike" and "sinelike" error present in the horizontal injection transient.

The longitudinal and horizontal response matrices are measured and singular value decomposition used to invert the matrix making sure both singular values are kept. Figure 6 shows the response matrix measurement data for the case of the longitudinal plane. The plots show the response of the real and imaginary parts of the frequencydomain oscillation at the synchrotron frequency $\Xi\left(\omega_{s}\right)$ to the "start ramp" time and booster rf phase actuators. The start ramp time determines the magnetic field the beam encounters at injection and therefore is used to correct energy errors $\Delta E$. The $\mathrm{rf}$ phase actuator can correct for $\mathrm{rf}$ phase errors $\Delta \phi$ due to both PAR and booster rf systems. Each longitudinal response matrix element indicated in Eq. (13) is proportional to the slope of the best-fit line of the corresponding plot in the figure. Horizontal response data are treated in an identical fashion.

Standard feedback software is used to perform the feedback [9]. The feedback software reads a two-component vector of real and imaginary FFT readbacks, multiplies this vector by the inverse response matrix, thereby obtaining a two-component vector of actuator changes. A fraction of the full actuator correction is applied by the software, and the process is repeated at an interval of 3 seconds. This interval allows plenty of time for waveform averaging of the transient, as well as averaging six samples of the real and imaginary readbacks.

Figure 7 shows progress of the longitudinal feedback system for a booster if phase error of $25^{\circ}$. The figure shows the real and imaginary parts of the FFT at the synchrotron tune peak along with the value of the booster rf phase actuator. The feedback algorithm converges after approximately 70 steps (210 seconds). The effect of noise on the real and imaginary FFT readbacks is reduced by allowing only a fraction of the full correction to be used (in this case $35 \%$ ). Noise on the readbacks becomes larger as they get closer to zero, since at this point the FFT peak is zero and the peak-finding algorithm records peaks randomly over the allowed peak-finding range. This noise, however, averages to zero with the result that the actuators move a small amount about their nominal setting.

Operation of the horizontal feedback is similar, as shown in Fig. 8. The figure shows an (artificial) abrupt change in the booster-injection kicker and septum voltage setpoints and the evolution of the feedback process. Similar to longitudinal feedback, the correction process converges after a few hundred steps for a three-second interval per step. Horizontal correction convergence takes longer because the feedback gain is reduced by half compared to the longitudinal feedback (15\% compared to 35\%). Reduced gain is required due to an increased amount of noise in the betatron signal compared to that for the longitudinal signal. Since only long-term drift is of concern, the increased 

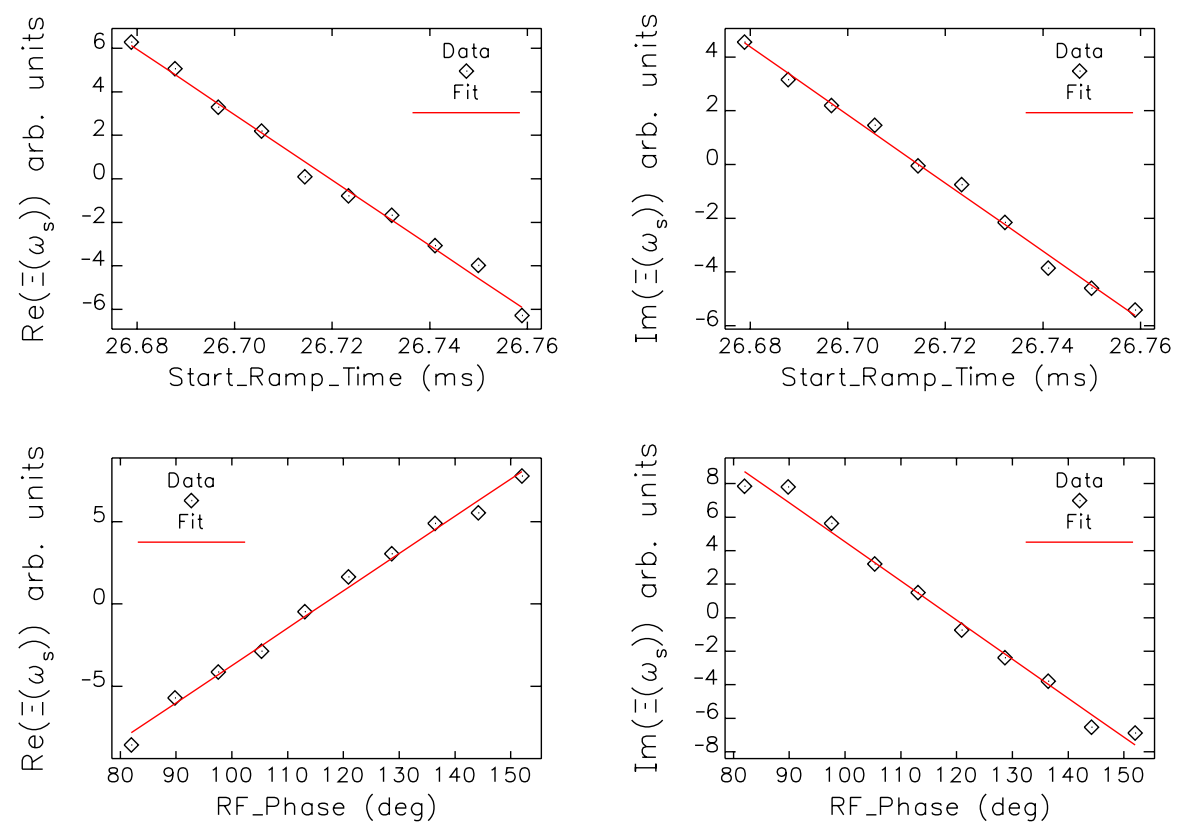

FIG. 6. (Color) Data used to determine the longitudinal response matrix $\mathbf{R}\left(\omega_{s}\right)$. The top two plots are the response of the real and imaginary parts of $\Xi\left(\omega_{s}\right)$ to the booster magnet "start ramp" time. The bottom two plots are the response of the real and imaginary parts of $\Xi\left(\omega_{s}\right)$ to the rf phase actuator of the booster rf system. Each longitudinal response matrix element in Eq. (13) is proportional to the slope of the best-fit line of the corresponding plot in the figure.

horizontal correction time is not an issue, because typical trajectory errors at injection require kicker and septum setpoint errors much smaller than those artificially produced in Fig. 8 (this statement is also true for longitudinal phase and energy errors).

Both longitudinal and horizontal transient feedback systems are optimized to correct slow drifts that occur on the order of tens of seconds to minutes. This allows time for averaging of the time-domain waveforms as well as the real and imaginary readbacks to reduce noise. Various test
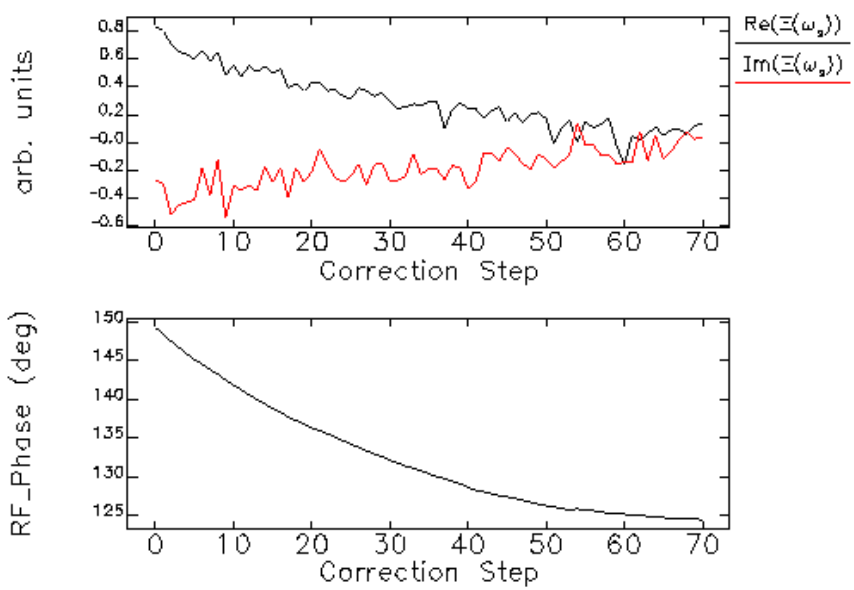

FIG. 7. (Color) Longitudinal feedback correction evolution for an rf phase error. The effect of noise on the real and imaginary FFT readbacks is reduced by allowing only a fraction of the full correction to be used (in this case $35 \%$ ). conditions are implemented that will stop the feedback from running if the test is not true. Test conditions include low beam charge, actuator variables near their limits, and booster magnet ramp parameters too far from nominal. It is important that the booster magnet ramps be well corrected so that the horizontal tune will be the same as the tune when the response matrix was measured. Both longitudinal and horizontal feedback systems have been in routine operational use in the APS booster since $2003[9,10]$. A
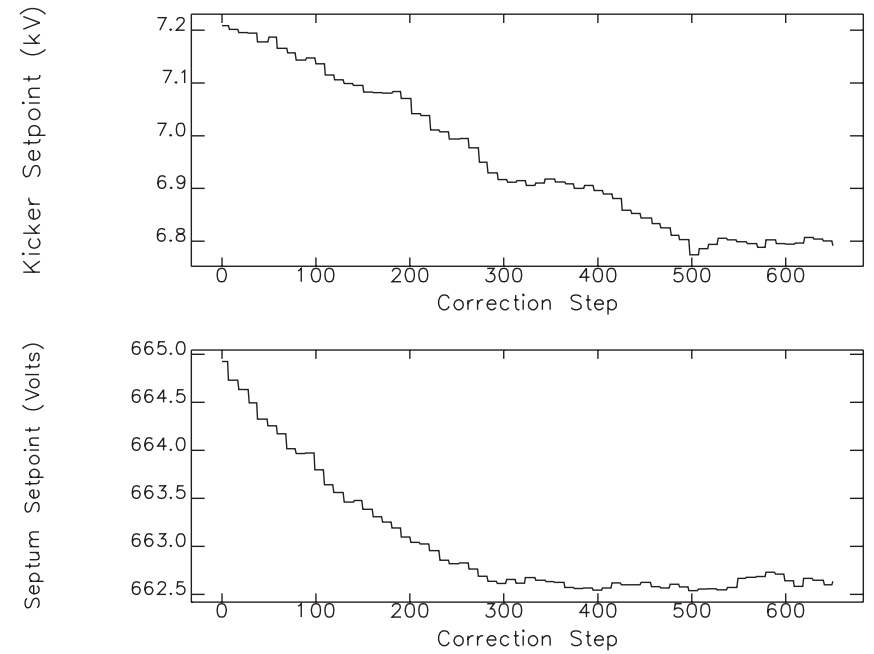

FIG. 8. Horizontal feedback correction evolution for kicker (top) and septum (bottom) errors. In this case $15 \%$ feedback gain is used. 
vertical feedback system using the same technique was commissioned but not implemented since the injected beam from the PAR is very stable vertically.

\section{CONCLUSION}

We have described feedback systems used to control the injected beam in the APS booster synchrotron. These feedback systems are based on measuring and processing the longitudinal synchrotron and horizontal betatron transient signals present at injection and performing feedback using actuators that correct energy, phase, horizontal position, and horizontal angle errors at injection. It was shown that the real and imaginary parts of the FFT at the synchrotron or horizontal betatron frequencies contain all the information needed to correct the transient using suitable actuator variables. In principle, this technique can be used when injecting into any circular accelerator provided that the injection transient signal can be detected. It therefore works very well in the APS booster synchrotron where only a single bunch is injected on axis every $2-\mathrm{Hz}$ cycle. In storage rings during top-up, a large stored beam current is present, and the BPM system has the additional requirement that it must detect the usually small injection transient signal in the presence of a large stored beam signal.

\section{ACKNOWLEDGMENTS}

The authors would like to thank Robert Lill for designing the upgraded booster BPM system electronics. Much appreciation also goes to Lester Erwin and Charles Gold for installing and maintaining the booster BPM electronics. Thanks to Michael Borland, Louis Emery, Glenn Decker, and Chih-Yuan Yao for their many useful comments and suggestions. This work was supported by the U.S. Department of Energy, Office of Science, Office of Basic Energy Sciences, under Contract No. DE-AC0206CH11357.

\section{APPENDIX A: FREQUENCY-DOMAIN COMPUTATION OF SYNCHROTRON AND BETATRON TRANSIENT MOTION}

Equations (9) and (10) describe transverse beam (transient) motion as discrete turn-by-turn data. We therefore take the DFT of the motion to obtain the transient motion in the frequency domain. The DFT of a sampled function $f\left(n T_{\text {o }}\right)$ is given by

$$
F\left(\omega_{m}\right)=\sum_{n=0}^{N-1} f\left(n T_{\circ}\right) e^{-j n \omega_{m} T_{\circ}},
$$

where the integer $m$ defines the frequency component of interest according to

$$
\omega_{m}=\frac{m}{N} \omega_{\circ},
$$

and $\omega_{\circ}=2 \pi / T_{\circ}$ is the sampling frequency, $N$ is the total number of samples or turns in our application, and $j=$ $\sqrt{-1}$ [3]. For our application the sampling frequency is identical to the booster revolution frequency. In effect, the BPM samples the betatron or synchrotron motion at the revolution frequency.

When evaluating the DFT analytically, it is convenient to write the synchrotron or betatron frequency in terms of an integer and fractional number of oscillations in the total time interval $N T$ 。 that sampling is performed. The synchrotron or betatron frequency can therefore be written as

$$
\omega_{\zeta}=\left(\frac{k_{\zeta}+\delta_{\zeta}}{N}\right) \omega_{\circ}
$$

where $\zeta$ refers to the longitudinal coordinate denoted by $s$ or the transverse coordinates denoted by $\xi ; k_{\zeta}$ is an integer and represents the number of complete oscillations in the sampling interval $N T_{\circ}$ and $\delta_{\zeta}$ is the "left over" fractional part of an oscillation in the sampling interval. For synchrotron oscillations where $\omega_{s} \ll \omega_{\circ}, k_{s}$ is significantly smaller than $N$, which means that synchrotron oscillations are oversampled. In the case of betatron oscillations, $k_{\xi}$ can be of the same order as the number of turns or samples $N$. Betatron oscillations can be undersampled or oversampled depending on the fractional part of the betatron tune. If the fractional part of the betatron tune is $\nu_{\xi}<0.5$, then the betatron oscillation is oversampled. If $0.5<\nu_{\xi}<1.0$, the betatron oscillation is undersampled. In the case of betatron undersampling, the information as to the type of error that caused the oscillation is still preserved at the Fourier component at the frequency corresponding to the fractional betatron tune.

Taking the DFT of Eqs. (9) and (10) at either the synchrotron or betatron frequencies amounts to being able to compute the DFT of the quantities $\cos \left(n \omega_{\zeta} T_{0}\right)$ and $\sin \left(n \omega_{\zeta} T_{\circ}\right)$. The DFT of these quantities is what appears in the response matrix equations (13) and (14). The result is

$$
\begin{aligned}
C\left(m, k_{\zeta}, \delta_{\zeta}\right)= & \sum_{n=0}^{N-1} \cos \left[2 \pi\left(k_{\zeta}+\delta_{\zeta}\right) / N\right] e^{-j 2 \pi n m / N} \\
= & \frac{1}{2} \sum_{ \pm} e^{j \pi\left(k_{\zeta}+\delta_{\zeta} \pm m\right)[(N-1) / N]} \\
& \times \frac{\sin \left[\pi\left(k_{\zeta}+\delta_{\zeta} \pm m\right)\right]}{\sin \left[\pi\left(k_{\zeta}+\delta_{\zeta} \pm m\right) / N\right]}, \\
S\left(m, k_{\zeta}, \delta_{\zeta}\right)= & \sum_{n=0}^{N-1} \sin \left[2 \pi\left(k_{\zeta}+\delta_{\zeta}\right) / N\right] e^{-j 2 \pi n m / N} \\
= & \frac{1}{2 j} \sum_{ \pm} \mp e^{j \pi\left(k_{\zeta}+\delta_{\zeta} \pm m\right)[(N-1) / N]} \\
& \times \frac{\sin \left[\pi\left(k_{\zeta}+\delta_{\zeta} \pm m\right)\right]}{\sin \left[\pi\left(k_{\zeta}+\delta_{\zeta} \pm m\right) / N\right]},
\end{aligned}
$$


where $m=k_{\zeta}$ is the frequency component closest to the true synchrotron or betatron frequency given by Eq. (A3). Only in the case where $\delta_{\zeta}=0$ does the single frequency component defined by $m=k_{\zeta}$ equal the exact synchrotron or betatron frequency. As expected, the ratio of sine functions in Eqs. (A4) and (A5) is nearly maximum at the synchrotron or betatron frequency. In many applications including ours, a specific time-domain window function $w\left(n T_{\circ}\right)$ is used to customize the spectrum in the frequency domain. In our application, we used a Blackman window, which has the effect of suppressing side lobes around the synchrotron and betatron frequencies. The results calculated in Eq. (A4) and (A5) used a simple rectangular window, which has significant sidelobes compared to the Blackman window [3].

[1] E. D. Courant and H. S. Snyder, Ann. Phys. (Leipzig) 3, 1 (1958).

[2] S. Y. Lee, Accelerator Physics (World Scientific, Singapore, 1999), pp. 218-219.
[3] R. G. Lyons, Understanding Digital Signal Processing (Addison-Wesley Longman, Inc., Reading, MA, 1997), pp. 121-122.

[4] S. V. Milton, in Proceedings of the 1995 Particle Accelerator Conference, pp. 594-596.

[5] J.A. Carwardine, S. V. Milton, and D. G. McGee, in Proceedings of the 1995 Particle Accelerator Conference, pp. 1967-1969.

[6] S. V. Milton and J. A. Carwardine, in Proceedings of the 1995 Particle Accelerator Conference, pp. 2708-2710.

[7] F. R. Lenkszus, E. Kahana, A. J. Votaw, G. A. Decker, Y. Chung, D. J. Ciarlette, and R. J. Laird, in Proceedings of the 1993 Particle Accelerator Conference, Washington, D.C., pp. 1814-1816.

[8] L. R. Dalesio, M. R. Kramer, and A. J. Kozubal, ICALEPS 1991.

[9] H. Shang, R. Soliday, L. Emery, and M. Borland, in Proceedings of the 2003 Particle Accelerator Conference, pp. 3470-3472.

[10] N.S. Sereno, Frank Lenkszus, and Robert M. Lill, in Proceedings of the 2003 Particle Accelerator Conference, pp. 2435-2437. 